\title{
DETERMINING KEY ENERGY RELATIONS, QUANTUM STATES AND ASSOCIATED MATRIX FORMALISMS RELATIVE TO THE COLLISION BETWEEN TWO DIATOMIC MOLECULES UNDER A STRONG MAGNETIC FIELD
}

\author{
M. A. Grado-Caffaro, M. Grado-Caffaro \\ M. A. Grado-Caffaro and M. Grado-Caffaro-Scientific Consultants, \\ C/Julio Palacios 11, 9-B, 28029-Madrid, Spain, \\ e-mail: ma.grado-caffaro@sapienzastudies.com \\ (Received - Febrary 18, 2019; in final form - September 23, 2019)
}

\begin{abstract}
We carry out a dynamical analysis of the collision process between two diatomic molecules in the presence of a strong magnetic field. In order to exemplify, these molecules can be, say, typically, lasing molecules as, for instance, relative to carbon-monoxide gasdynamic lasers. The molecules in question behave as quantum anharmonic oscillators after the Morse potential. In our analysis, we simulate the kinetic energy lost after the collision by assuming, equivalently, two additional molecules (fictitious and identical to the molecules which collide) with the above kinetic energy. Furthermore, the corresponding density of states is determined and associated matrix formalisms are established to calculate the total number of states.

Key words: diatomic molecules, collision processes, morse potential, magnetic field, lost kinetic energy, density of states.
\end{abstract}

DOI: https://doi.org/10.30970/jps.23.4002

PACS number(s): 02.10.Yn, 03.65.-w, 33.20.Tp, 34.20.-b, 34.50.-s

\section{INTRODUCTION}

The Morse-potential model plays an important role in Molecular and Nuclear Physics. In fact, a number of phenomena of the aforementioned fields can be modelled by introducing a Morse-type potential. In particular, in Atomic and Molecular Physics as well as in Condensed Matter Physics, we may find relevant examples in which the above type of potential is suitable to describe quantitatively the involved anharmonic-oscillator behaviour [1-6]. As a matter of fact, we may consider certain disordered systems in which electrons behave as anharmonic oscillators under the action of a Morse potential. In addition, under certain conditions, the atoms of a distorted or even ordered crystalline lattice behave as anharmonic oscillators submitted to a Morse-type potential. On the other hand, the vibrational behaviour of diatomic molecules can be modelled by the Morse potential. By the way, after the united-atom approximation (see, for instance, Ref. [7]), the vibrational motion of some polyatomic molecules can be also modelled by the Morse approach.

The aim of the present article is developing a theoretical formulation to explain the collision process between two diatomic molecules that are under an intense magnetic field. For instance, in relation to high-power lasers (particularly, gasdynamic lasers), one may consider collision processes in the context of vibrational behaviour of carbon-monoxide molecules or nitrogen molecules, which play an important role as lasing molecules $[8,9]$. Other typical examples deal with certain chemical reactions for the formation of transition-metal oxides $[10,11]$ as $\mathrm{CdO}$ and $\mathrm{ZnO}$. As a matter of fact, we will present a dynamic study on the aforementioned collision process. Particularly, in this study, we will elaborate, say, an equivalence scheme to simulate the kinetic energy lost after the collision by means of two additional (fictitious) diatomic molecules (identical to the molecules that collide) with the above mentioned kinetic energy. Moreover, the corresponding density of states will be calculated and associated matrix formalisms will be presented.

\section{THEORETICAL FORMULATION}

Let us consider a diatomic molecule under the action of a one- dimensional Morse potential. The corresponding potential energy can be expressed as follows:

$$
V(x)=D\left\{1-\exp \left[-\alpha\left(x-x_{0}\right)\right]\right\}^{2},
$$

where $x$ is cartesian coordinate in the direction of the inter-nuclear axis of the molecule, $D$ is the potential depth, $x_{0}$ is the equilibrium position of the molecule, and $\alpha$ is a parameter associated directly with the width of the potential. The corresponding non-relativistic, timeindependent, Schrödinger equation for a molecule of mass denoted by $m$, reads:

$$
\frac{d^{2} \psi_{n}(x)}{d x^{2}}+\frac{2 m}{\hbar^{2}}\left[E_{n}-V(x)\right] \psi_{n}(x)=0
$$

where the quantum number $n$ will be defined later. It is well-known that the total energy eigenvalues of Eq. (2) with formula (1) are given by the following relationship, which can be considered as a two-variable functional se- 


\section{A. GRADO-CAFFARO, M. GRADO-CAFFARO}

quence as follows:

$$
E_{n}(\omega, \varepsilon)=\hbar \omega\left(n+\frac{1}{2}-n \varepsilon-n^{2} \varepsilon-\frac{\varepsilon}{4}\right),
$$

where $\omega$ denotes the fundamental angular frequency of the anharmonic oscillator in question, $n=0,1,2, \ldots$ is the corresponding vibrational quantum number, and $\varepsilon$ is a relatively small anharmonic coefficient. Parameters $\omega$ and $\varepsilon$ satisfy $\varepsilon=h \omega /(4 D)$; so one has three parameters, namely, $\omega, \varepsilon$ and $D$, two of them being mutually independent. Therefore, we can take $\omega$ and $\varepsilon$ as mutually independent parameters which, regarding all the possible Morse oscillators, may be viewed as real variables. At this point, we recall that Eq. (3) represents a realvalued functional sequence with two real variables.

We regard two diatomic molecules initially in the presence of a perpendicular intense magnetic field, so (initially) the molecules move circularly. Assume that, later, the field acts in the direction of the inter-nuclear axis of the molecules so that we are interested in the vibrational behaviour of the molecules in question under the potential energy represented in Eq. (1). So, the molecules behave as quantum anharmonic oscillators. Assume also that the molecules collide due, for instance, to photon interaction. In this context, a, say, typical example is lasing molecules as we have said in Section 1. Before the collision, we suppose that the two molecules are in state $n$. After they collide (consider, for example, intense laser interaction), one of them is pumped up to state $n+1$ and the other molecule falls down to state $n-1$ (note that, of course, $n \geq 1$ ) [8,9]. Therefore, under all the above conditions, it follows:

$$
2 E_{n}-E_{n+1}-E_{n-1}=E_{\text {kin }},
$$

where $E_{\text {kin }}$ is kinetic energy resulting from that $\varepsilon \neq 0$. Note that $E_{\text {kin }}=0$ if $\varepsilon=0$ (harmonic case). Combining (3) with (4), one gets:

$$
E_{\text {kin }}=2 \hbar \omega \varepsilon \text {. }
$$

On the other hand, we have that $\omega=e B / m$ (cyclotron angular frequency) where $B$ denotes the induction (magnetic flux density) of the involved magnetic field and $e$ is the absolute value of the electron charge. Although the above field is strong enough (so $\omega$ may be relatively high), $\varepsilon$ can have, say, moderate values (less than $1 / 2$ ) because $\varepsilon=\hbar \omega /(4 D)$ and $D$ should be large enough. At this point, notice that $\varepsilon \rightarrow 0$ when $D \rightarrow \infty$ (for not too large $\omega)$. On the other hand, formula (3) with $\varepsilon=0$ and $\omega=e B / m$ corresponds to the so-called Landau quantum states, whereas, considering that $\varepsilon \neq 0$, we may speak of Morse quantum states [6], that is, from the formulation of the present paper. It is clear that this formulation is a generalization of the Landau approach.

The fact that there is lost kinetic energy [see Eq. (5)] suggests simulating this fact by introducing two additional diatomic molecules identical to the former ones (after the molecular collision in question) with the above kinetic energy. We emphasize that, in addition to the two real molecules with respective states $n+1$ and $n-1$ after the collision, we introduce two fictitious molecules (identical to the former ones) of the kinetic energy given by formula (5). This may be viewed as an equivalence theorem. Thus, we have that $E_{\text {kin }}=m_{r} v^{2} / 2$ where $m_{r}$ is the reduced mass of the two fictitious molecules and $v$ is the magnitude of the velocity of the molecule center of mass; one has that $m_{r}=m / 2$. Combining these facts with formula (5) and with the above expressions for $\varepsilon$ and $\omega$, then we have:

$$
v=\frac{\hbar e B}{m} \sqrt{\frac{2}{m D}} .
$$

Looking at formula (6), notice that $v$, although been proportional to $B$ (which is strong), is inversely proportional to $D$ ( $D$ is relatively large), so $v$ is moderate. On the other hand, note the significant mass-dependence of $v$. Now we are interested in determining the associated density of states. To achieve this end, we will consider both atomic and molecular orbitals in the context of Refs. [12-14]. Within this framework, an accurate expression for the density of states of each molecule before the molecular collision is:

$$
g(E)=\sum_{n, k}\left|\psi_{n} * \varphi_{k}\right|^{2} w_{k}(E) \delta\left(E-E_{n}\right),
$$

where $\varphi_{k}$ stands for atomic orbitals (electron wavefunctions), the asterisk denotes convolution, $w_{k}(E)$ are weight functions, and $\delta$ designates Dirac's delta function. We remark the role of $\psi_{n} * \varphi_{k}$ as quantum anharmonic oscillator strength. On the other hand, notice that $n, k=1,2, \ldots$. Notice also that including weighted quantities into Eq. (7) is a relevant fact whose meaning is clear: formula (7) is an actually accurate expression which improves the state of the art, providing useful information that is missing fron other formulations. After the molecules collide, one considers [in Eq. (7)], respectively, $n+1$ and $n-1$ instead of $n$. The total number of quantum states reads:

$$
N=\int_{0}^{\infty} g(E) d E
$$

Replacing (7) into (8), it follows (before the collision):

$$
N=\sum_{n, k}\left|\psi_{n} * \varphi_{k}\right|^{2} w_{k}\left(E_{n}\right) .
$$

By defining the matrix elements, namely, $\left|\psi_{n} * \varphi_{k}\right|^{2} \equiv$ $\gamma_{n k}$ and $w_{k}\left(E_{n}\right) \equiv u_{n k}$, relation (9) becomes an inner (dot) product of matrices as follows:

$$
N=\Gamma \bullet U
$$

where $\Gamma=\left(\gamma_{n k}\right)$ and $U=\left(u_{n k}\right)$. Relation (9) can be also viewed as follows:

$$
N=\operatorname{tr}\left(\Gamma^{t} U\right)
$$

where $t r$ denotes trace. Relations (10) and (11) constitute matrix formalisms for expressing the total number of 
states (before the collision) in an elegant and concise way. After the collision, straightforwardly similar results are obtained. Another interesting formalism reads [13-16]:

$$
N=\operatorname{tr}\left(\Gamma^{t} \otimes U\right)
$$

where the tensorial product is not the tensor product in the Kronecker sense used in standard Quantum Mechanics but it is a dyadic-tensor product as in the following example relative to $2 \times 2$ matrices:

$$
\left(\begin{array}{ll}
a & b \\
c & d
\end{array}\right) \otimes\left(\begin{array}{ll}
a^{\prime} & b^{\prime} \\
c^{\prime} & d^{\prime}
\end{array}\right)=\left(\begin{array}{llll}
a a^{\prime} & a b^{\prime} & a c^{\prime} & a d^{\prime} \\
b a^{\prime} & b b^{\prime} & b c^{\prime} & b d^{\prime} \\
c a^{\prime} & c b^{\prime} & c c^{\prime} & c d^{\prime} \\
d a^{\prime} & a b^{\prime} & d b^{\prime} & d d^{\prime}
\end{array}\right)
$$

Note that the product in question of expression (13) is non-commutative. In fact, the transpose of the righthand side of (13) equals the left-hand side with changing the order of the matrices. By virtue of this, formula (12) becomes:

$$
N=\operatorname{tr}(U \otimes \Gamma)^{t}
$$

On the other hand, by using the Hadamard-Schur product of matrices (that is, multiplying homologous matrix elements), one has:

$$
\Gamma \circ U=\left(\gamma_{n k} u_{n k}\right)
$$

Therefore, the usual norm of the above product written in formula (15) reads:

$$
\Gamma \circ U=\sum_{n, k} \gamma_{n k} u_{n k}
$$

By formulae (9) to (13), it follows:

$$
N=\Gamma \circ U=\Gamma \bullet U=\operatorname{tr}\left(\Gamma^{t} U\right)=\operatorname{tr}(\Gamma \otimes U)=\operatorname{tr}(U \otimes \Gamma)^{t}
$$

\section{DISCUSSION AND CONCLUSIONS}

For the first time, we have presented an accurate analysis of an interesting molecular collision process within the framework of the Morse potential associated with the presence of a strong magnetic field. It is clear that the involved molecules are quantum anharmonic oscillators [6] that can be, for example, molecules under an intense laser field. Since molecules as quantum harmonic oscillators under a magnetic field occupy the so-called Landau states, then one may say that molecules (or particles in general) as Morse (quantum) oscillators under a magnetic field occupy the Grado-Caffaro states [6]. Unfortunately, not much has been done, to date, with regard to quantum anharmonic oscillators. At this point, we should claim that, certainly, anharmonicity occupies a notorious place in the context of Nonlinear Physics. One must remark especially that our study is unprecedented as it opens up new perspectives on how to tackle similar problems from a strict mathematical-physics standpoint. Our collision process has been described mathematically with significant rigour. In particular, formula (6) is a direct consequence of the equivalence theorem by which four identical molecules are involved. The key points of the present article are formulas (4) to (9), which arise from our analysis of the involved density of states, and (10) to (17) relative to matrix formalisms concerning the total number of states. Both mathematical elegance and usefulness come from these formalisms. On the other hand, we would note the importance of the concept of density of states in various areas of Physics. For instance, we may refer to the integrated density of states for a periodic Schrödinger operator (Hamiltonian operator for the Schrödinger equation with periodic potential) [19-22].
[1] J. A. Coxon, P. G. Hajigeorgiou, J. Chem. Phys. 132, 094105 (2010); https://doi.org/10.1063/1.3319739.

[2] R. J. Le Roy, Y. Huang, C. Jary, J. Chem. Phys. 125 164310 (2006); https://doi.org/10.1063/1.2354502.

[3] N. S. Dattani, R. J. Le Roy, J. Mol. Spectrosc. 268 199 (2013); https://doi.org/10.1016/j.jms.2011.03. 030.

[4] L. K. Grover, Phys. Rev. Lett. 79, 325 (1997); https: //doi.org/10.1103/PhysRevLett.79.325.

[5] S. A. Mikhailov, Physica B 299, 6 (2001); https://doi. org/10.1016/S0921-4526(00)00769-9.

[6] M. A. Grado-Caffaro, M. Grado-Caffaro, Eur. Phys. J. B 73, 243 (2010); https://doi.org/10.1140/epjb/ e2009-00412-0.
[7] S. Y. Bhide, S. Yashonath, J. Phys. Chem. A 106, 7130 (2002); https://doi.org/10.1021/jp002626h.

[8] R. L. McKenzie, Phys. Fluids 15, 2163 (1972); https: //doi.org/10.1063/1.1693852.

[9] J. D. Anderson, jr., Gasdynamic lasers: an introduction (Academic Press, New York, 1976), p. 113.

[10] A. A. Dakhel, Curr. Appl. Phys. 11, 11 (2011); https: //doi.org/10.1016/j.cap.2010.06.003.

[11] Tz-Jun Kuo, M. H. Huang, J. Phys. Chem. B 110, 13717 (2006); https://doi.org/10.1021/jp062854x.

[12] J. Owen, J. H. M. Thornley, Rep. Prog. Phys. 29, 675 (1966); https://doi.org/10.1088/0034-4885/29/ $2 / 306$.

[13] G. Amat, H. H. Nielsen, G. Tarrago, Rotation-vibration 
of polyatomic molecules (Marcel Dekker, Inc., New York, NY, 1971).

[14] M. Dang-Nhu, A. S. Pine, Can. J. Phys. 64, 289 (1986); https://doi.org/10.1139/p86-051.

[15] M. A. Grado-Caffaro, M. Grado-Caffaro, Mod. Phys. Lett. B 18, 1449 (2004); https://doi.org/10.1142/ S0217984904007918.

[16] M. A. Grado-Caffaro, M. Grado-Caffaro, Rep. Math. Phys. 70, 105 (2012); https://doi.org/10.1016/ S0034-4877 (13)60015-4.

[17] M. A. Grado-Caffaro, M. Grado-Caffaro, Adv. Stud. Theor. Phys. 8, 73 (2014); https://doi.org/10.12988/ astp. 2014.311137.
[18] M.A. Grado-Caffaro, M. Grado-Caffaro, Z. Naturforsch. A 72, 463 (2017); https://doi.org/10.1515/ zna-2016-0485.

[19] B. E. J. Dahlberg, E. Trubowitz, Comment. Math. Helvetici 57, 130 (1982); https://doi.org/10.1007/ BF02565850.

[20] A. Mohamed, J. Math. Phys. 38, 4023 (1997); https: //doi.org/10.1063/1.532105.

[21] M. M. Skriganov, Inv. Math. 80, 107 (1985); https: //doi.org/10.1007/BF01388550.

[22] A. V. Sobolev, Ann. Henri Poincaré 6, 31 (2005); https: //doi.org/10.1007/S00023-005-0198-8.

\title{
ВИЗНАЧЕННЯ КЛЮЧОВИХ ЕНЕРГЕТИЧНИХ СПІВВІДНОШЕНЬ, КВАНТОВИХ СТАНІВ ТА ПОВ'ЯЗАНИХ 3 НИМИ МАТРИЧНИХ ФОРМАЛІЗМІВ, ЩО СТОСУЮТЬСЯ ЗІТКНЕННЯ ДВОХ ДВОАТОМНИХ МОЛЕКУЛ У СИЛЬНОМУ МАГНІТНОМУ ПОЛІ
}

\author{
М. А. Ірадо-Каффаро, М. Ірадо-Каффаро \\ мадрид, Іспанія
}

Проведено динамічний аналіз процесу зіткнення двох двоатомних молекул за наявності сильного магнітного поля. Це можуть бути, наприклад, молекули в газодинамічних лазерах на основі оксиду вуглецю. Молекули, про які йдеться, поводяться як квантові ангармонічні осцилятори в потенціалі Морзе. У нашому аналізі, вводячи дві додаткові фіктивні молекули (ідентичні молекулам, що стикаються) із такою самою кінетичною енергією, моделюємо втрату кінетичної енергїі внаслідок зіткнення. Крім того, визначаємо відповідну густину станів і встановлюємо пов'язані матричні формалізми для обчислення повної кількості станів. 